\title{
The left-digit effect in price and date perception
}

\author{
Joey Tang ${ }^{1,2 *}$
}

\begin{abstract}
Research on consumer behaviour suggests that people perceive nine-ending prices (e.g. \$2.99) to be significantly lower than prices one cent higher (\$3.00). This is known as the left-digit effect. This effect was replicated in a group of Chinese participants residing in Hong Kong where nine-ending prices such as $\mathbf{\$ 2 . 9 9}$ do not occur in the local pricing system, suggesting that familiarity of the pricing format has little influence on the effect. A novel finding was also reported with the left-digit effect observed in the domain of date perception. Participants rated lastday-ending dates such as 5.31 (representing May 31) as significantly earlier than first-day-ending dates such as 6.01 (June 1). Price and date judgements are juxtaposed against one another and discussed in terms of the rounding-up aversion account. Potential pitfalls in consumer behaviour are also highlighted.
\end{abstract}

Keywords: left-digit effect, consumer behaviour, price perception, date perception

\section{INTRODUCTION}

Research on consumer behaviour suggests that people perceive nine-ending prices to be significantly lower than prices one cent higher (1). However, not all nine endings affect price perception. Only those involving a left digit change will be perceived as significantly cheaper. For example, $\$ 2.99$ is perceived as significantly cheaper than $\$ 3.00$, but not when comparing $\$ 3.59$ against $\$ 3.60$. The former price pair contains different left digits, leading to a larger perceived difference. This phenomenon has been termed the left-digit effect (1). The effect has since been replicated in a number of studies on price perception and is not only robust with lower-priced products (2) but also manifests with higherpriced transactions observed in the housing market (3). In the latter study, similar properties listed with smaller left digits compared to larger left digits (of only $\$ 100$ apart) were more likely to sell and were on the market for a fewer number of days. These studies clearly demonstrated a strong bias towards to the left digit(s) with astonishing behavioural consequences.

Consumers are not the only ones who display the left-digit effect; investors' perception of profit has also been shown to be disproportionately affected by the leftmost digit (4). In a study which examined over 7 million trades made by over 25,000 individuals, a strong left-digit effect was observed, indicating that investors focused more on the left digit(s) in profit than price when deciding when to close a trade. This bias could potentially lead to the dire consequence of losing billions of dollars per financial year for investors.

According to Thomas and Morwitz (2005), the left-digit effect is domain-invariant and manifests also with non-price stimuli (1). Indeed, the effect has been observed outside monetary terms, for example, when rating product quality (1). Similarly, when judging school performance in terms of numerical information, a strong leftdigit effect was observed while the right (decimal) digit was largely ignored (5).

Building upon Dehaene's (1997) analogue model (6), Thomas and Morwitz (2005) explained the left-digit effect in terms of a primacy effect exerted by the leftmost digit during the conversion from numerical symbols to mental magnitudes (1). Since we read numbers from left to right, the magnitude encoding process for the price "2.99" occurs as soon as our eyes encounter the digit "2". Consequently, the encoded magnitude of $\$ 2.99$ gets anchored on the leftmost digit, i.e. $\$ 2$ and becomes perceived as significantly

\footnotetext{
${ }^{1}$ Center for Language and Brain, Shenzhen Institute of Neuroscience, Shenzhen China. ${ }^{2}$ Myndful Learning Association, Hong Kong SAR, China.

*Corresponding author. Email: joey.tang@uclmail.net (J.T.)
}

lower than the encoded magnitude of $\$ 3.00$ (i.e. an encoding bias). It is the dollar digit difference rather than the actual price difference of one cent that produces the distorted perception. The left-to-right processing account - which assumes that the leftmost digit is read first and remembered more vividly - is supported by Coulter's research (7). However, findings from another study suggest that adults process the decade- and unit-digits in two-digit whole numbers in a parallel fashion rather than sequentially from left to right $(8)$. More recent research suggests that the left-digit effect arises from an aversion to rounding-up (as opposed to an encoding bias) in order to preserve the visual integrity of magnitude information (9).

In Hong Kong, the smallest price unit is ten cents. Thus, nine-ending prices like $\$ 2.99$ are unfamiliar to the locals. In Experiment 1, we examined whether the left-digit effect would be observed in the local community by replicating Thomas and Morwitz's (2005) study (1). In Experiment 2, we attempted to further investigate the domain-invariant property of the left-digit effect by replicating the aforementioned experiment in the domain of date perception. Unlike product quality rating (1) and school performance judgement (5), date perception does not bear any magnitude information but merely ordinal/sequential information, thus serves as a more appropriate domain for investigation.

\section{RESULTS}

For each experiment, a 2 x 2 mixed measures ANOVA was conducted on the difference in subjective ranking between the target and the standard, with condition (experimental or control) as the within-participants factor and ending (nine-ending or zero-ending in Experiment 1; last-day-ending or first-day-ending in Experiment 2) as the between-participants factor. A significant main effect of condition was found in both experiments (in Experiment 1, $F(1$, $30)=38.70, p<.001$; in Experiment $2, F(1,30)=15.38, p<.001)$. In both experiments, a significant condition $\mathrm{x}$ ending interaction was observed (in Experiment $1, F(1,30)=8.24, p<.05$; in Experiment $2, F(1,30)=9.30, p<.05)$. Further analyses showed that in Experiment 1, the subjective difference between nine-ending prices and the standard was significantly larger than that between zero-ending prices and the standard in the experimental condition $(t(30)=-2.16, p<.05)$ but not the control condition $(t(30)<$ 0.001, n.s.) (see Fig. 1). Likewise in Experiment 2, the subjective difference between late-date-ending birthdays and the standard was significantly larger than that between first-day-ending birthdays and the standard in the experimental condition $(t(26)=-2.03, p<.05)$ but not the control condition $(t(30)=-0.70, n . s$.) (see Fig. 2). These 
findings suggest that our perception is significantly affected by the leftmost digit in both price and date judgements.

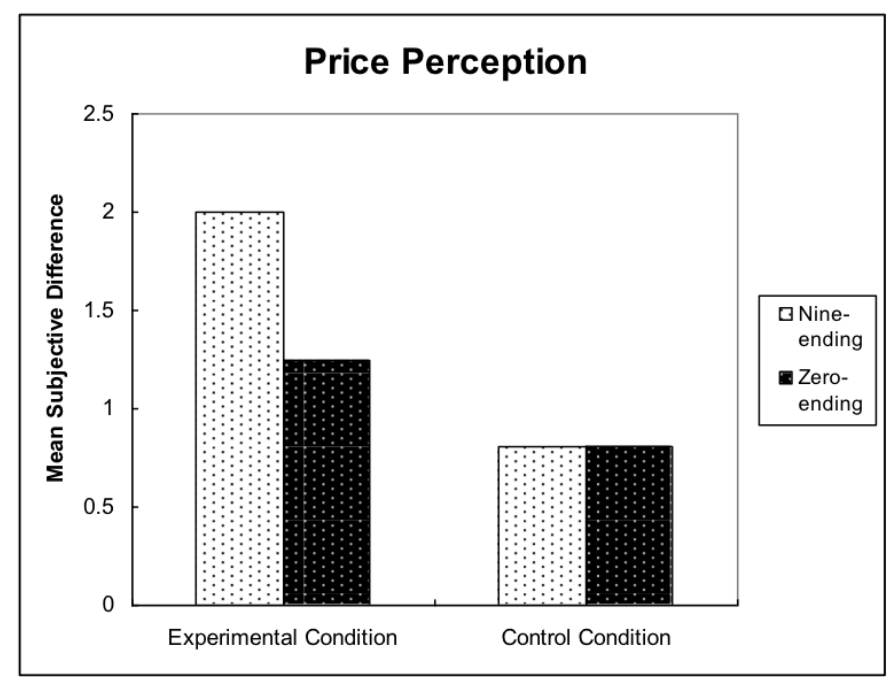

Fig. 1. Mean subjective difference between the target and the standard in price perception.

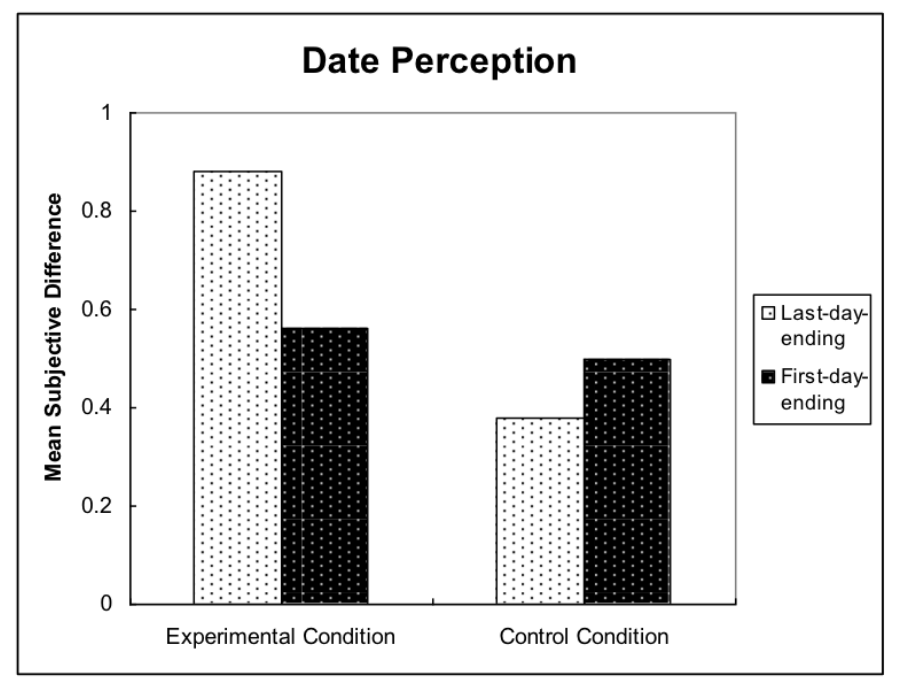

Fig. 2. Mean subjective difference between the target and the standard in date perception.

\section{DISCUSSION}

The left-digit effect in price judgement is a robust phenomenon and has a remarkable impact on consumer behaviour. In Experiment 1, we replicated the left-digit effect with Chinese participants in Hong Kong. Nine-ending prices such as \$2.99 do not occur in the local pricing system as the smallest unit is ten cents, yet participants perceived such prices as significantly cheaper than prices that are one cent higher (e.g. \$3.00). This finding suggests that familiarity with nine-ending prices is not important for the manifestation of the left-digit effect.

There is no doubt that the left-digit effect is robust in the pricing domain with powerful behavioural consequences. As aforementioned, the left-digit effect influences decisions of both consumers and investors $(3,4)$, sometimes with disastrous consequences potentially leading to huge losses. Not only do consumers and investors need to be educated to avoid pitfalls that could potentially arise from the left-digit bias but individuals should also be taught to distinguish between numerical distance and psychological (or perceived) distance in order to function as rational decision makers.

Results from Experiment 2 demonstrated, for the first time, the left-digit effect in the domain of date perception - a dimension consisting of no magnitude information but merely ordinal/sequential information. Participants perceived last-day ending dates (e.g. 5.31 representing May 31) as significantly earlier than dates that were one day later (e.g. 6.01 representing June 1). Although previously demonstrated in non-price domains, the left-digit effect that manifested in product quality rating $(l)$ and school performance perception (5) is not in essence different from the left-digit effect observed in price perception; judgements on these dimensions both involve magnitude information (i.e. making higher/lower or larger/smaller judgements). Date judgements, in contrast, concern ordinal/sequential but not magnitude information, i.e. making earlier/later or before/after judgements. Thus, date perception serves as a more appropriate domain to test the domain-invariant property of the left-digit effect.

The left-digit effect observed in date perception has an important practical application. Since participants perceived last-day ending dates (e.g. May 31) as significantly earlier than first-day ending dates (e.g. June 1), it would make more practical sense to set deadlines on last-day ending dates. For example, setting the deadline for a term paper on May 31 would prompt students to think that the deadline is much earlier than one set on June 1, thus encouraging them to work on and finish their assignment on time. This hypothesis, of course, warrants further investigation.

The current novel finding of a left-digit effect in the domain of date perception is an intriguing one. While there is no way to accurately determine the underlying cognitive mechanism of the left-digit effect from results of the present study, the effect observed in date perception points us into an interesting direction when considering the rounding-up aversion account. Since date as a dimension does not contain any magnitude information, it seems inappropriate or even nonsensical to discuss the effect in terms of the rounding-up aversion account. In reality, we rarely/never consider May 31 part of June, thus we do in effect avoid "roundingup" in this domain in order to preserve the visual integrity of the information.

In summary, the current findings provide an important extension and affirmation of the domain-invariant property of the left-digit effect. Although the underlying cognitive mechanism of the effect requires further investigation, the present study highlights the importance of educating people to avoid falling for pitfalls that could potentially arise from the left-digit bias.

\section{MATERIALS AND METHODS}

\section{Experiment 1 \\ Participants}

Thirty-two students (14 males and 18 females), aged between 18 and 23 years $(M=20.59$; S.D. $=1.29)$, from different universities in Hong Kong took part in Experiment 1. All of them were native Cantonese speakers.

\section{Stimuli and Design}

The method used in Experiment 1 represented an exact replication of the design employed by Thomas and Morwtz (2005) (1). Participants were presented with eight stationery price pairs; there were four different pairs, each presented twice (see Table 1 for the 
stimulus set). Each pair contained a target price and a fixed standard (\$4.00). The target was either a nine-ending price (e.g. \$2.99) or a zero-ending price (e.g. \$3.00). In the experimental condition, the nine- and zero-ending pairs contained different left digits (e.g. " 2 " and " 3 " in $\$ 2.99$ and $\$ 3.00$ respectively), whereas in the control condition, the nine- and zero-ending pairs contained the same left digit (e.g. \$3.59 and \$3.60). In each trial, participants had to give a subjective rating on a five-point Likert scale as to whether they agreed with the statement that the price of the stationery, either the target or the standard, was high.

Table 1. Stimulus set of Experiment 1 on price perception.

\begin{tabular}{ccccc}
\hline & \multicolumn{2}{c}{ Experimental } & \multicolumn{2}{c}{ Control } \\
\hline & Nine-ending & Zero-ending & Nine-ending & Zero-ending \\
\hline Target & 2.99 & 3.00 & 3.59 & 3.60 \\
\hline Standard & 4.00 & 4.00 & 4.00 & 4.00 \\
\hline
\end{tabular}

\section{Experiment 2}

\section{Participants}

For Experiment 2, a different group of 32 native Cantonesespeaking university students (18 males and 14 females) from Hong Kong were recruited. The age range was 19 to 23 years $(M=20.97$; S.D. $=1.23)$.

\section{Stimuli and Design}

The design of Experiment 2 followed closely that of Experiment 1, except that the domain concerned was now date rather than price perception. Participants were shown a total of eight birthday pairs (four different pairs; each presented twice) and were asked to give, for each pair, a subjective rating as to whether they agreed with the statement that the birthday, either the target or the standard, was late (see Table 2 for the stimulus set). Each pair contained a target date and a fixed standard (7.01, representing July 1). The target ended either with the last day of the month (last-day- ending, e.g. 5.31 representing May 31) or with the first day of the month (first-day-ending, e.g. 6.01 representing June 1). In the experimental condition, the last-day- and first-day-ending pairs contained different left digits (e.g. "5" and " 6 " in 5.31 and 6.01 respectively), whereas in the control condition, the last-day- and first-day-ending pairs contained the same left digit (e.g. 6.19 and 6.20).

Table 2. Stimulus set of Experiment 2 on date perception.

\begin{tabular}{ccccc}
\hline & \multicolumn{2}{c}{ Experimental } & \multicolumn{2}{c}{ Control } \\
\hline & Last-day-ending & First-day-ending & Last-day-ending & First-day-ending \\
\hline Target & 5.31 & 6.01 & 6.19 & 6.20 \\
\hline Standard & 7.01 & 7.01 & 7.01 & 7.01
\end{tabular}

\section{REFERENCES}

1. M. Thomas, V. Morwitz, Penny wise and pound foolish: The left-digit effect in price cognition J. Consum. Res. 32, 54-64 (2005).

2. C. Lin, J. Wang, Distortion of price discount perceptions through the left-digit effect. Mark. Lett. 28, 99-112 (2017).

3. S. Chava, V. W. Yao, Cognitive reference points, the left-digit effect, and clustering in housing markets. SSRN Electronic Journal (2017).

4. A. P. Kansara, M.-C. Sung, T. Ma, J. E. V. Johnson, Towards a better understanding of the ful impact of the left digit effect on individual trading behaviour: Unearthing a trading profit effect. Eur. J. Finance 26, 874-891 (2020).

5. A. L. Olsen, Leftmost-digit-bias in an enumerated public sector? An experiment on citizens' judgment of performance information. Judgm. Decis. Mak. 8, 365-371 (2013).

6. S. Dehaene, The Number Sense (New York; Oxford, 1997).

7. K. S. Coulter, Odd-ending price underestimation: An experimental examination of left-to-right processing effects. J. Prod. Brand Manag. 10, 276-292 (2001).

8. D. Ganor-Stern, J. Tzelgov, R. Ellenbogen, Automaticity and two-digit numbers. J. Exp. Psychol. Human 33, 483-496 (2007).

9. M. Thomas, T. Sokolova, Focusing on the left digit: An encoding or an estimation bias? Adv. Consum. Res. 42, 690-691 (2014).

Acknowledgments: The author gratefully acknowledges the support of Miss Shun Chi Leung, Miss Kar Hei Dawn Ying and Miss Wai Man Li for their assistance on data collection and analysis and Dr Winnie Wai Lan Chan for her contribution to an earlier version of this manuscript. Funding: This study was self-funded. Author contributions: Dr Joey Tang contributed to the experimental design, data interpretation and preparation of the manuscript. Competing interests: The author declares no competing interests, financial or otherwise, in completion of this work. Data and materials availability: All data and materials are available are upon request to the corresponding author. 